\title{
Multilayer krypton phase diagram
}

\author{
Peter Day, Marissa LaMadrid, Mark Lysek, and David Goodstein \\ Condensed Matter Physics 114-36, California Institute of Technology, Pasadena, California 91125
}

(Received 12 August 1992)

\begin{abstract}
We present vapor pressure and high-resolution heat-capacity measurements of multilayer krypton films adsorbed on graphite. We have found a complex phase diagram including reentrant layering transitions for coverages higher than three layers. The third layering transition does not end in a condensation critical point as was previously expected. The second-layer melting transition is preceded by what may be a commensurate-incommensurate transition between the first and second layers.
\end{abstract}

\section{INTRODUCTION}

Recent studies of adsorbed films have revealed extremely complex multilayer phase diagrams. ${ }^{1}$ The firstorder layering transitions, by which a film grows at low temperature, were expected, based on several calculations, ${ }^{2}$ to end in layer condensation critical points associated with the roughening of the bulk surface. Ellipsometry ${ }^{3}$ studies on argon, krypton, and xenon films have shown that new layering transitions appear at higher temperatures, shifted in coverage and chemical potential. A vapor pressure study has confirmed this behavior for the case of argon films. ${ }^{4}$ These new reentrant layering transitions are believed to be due to the coexistence between films with surface occupancy of about half a layer. Our own heat-capacity study of argon films ${ }^{5}$ has further shown that the low-temperature and reentrant layering transitions are joined together by heat-capacity peaks suggesting additional phase transitions. It has been conjectured that the reentrant layering phenomenon may be related to a preroughening transition at the bulk interface. $^{6}$

The phase diagrams of the first few layers of a multilayer film are different from those of higher layers, because the surface of a very thin film does not closely resemble a crystal surface. Part of the reason for the difference is that the first few layers are affected by the strong Van der Waals potential of the substrate. On the other hand, a model taking into account the stacking of the atoms of the film, called the fcc adsorption model ${ }^{7}$ by Saam, further predicts that the first- and second-layer phase diagrams should be topologically different from those of the higher layers. This model assumes that each layer acts as a lattice gas and forms a commensurate structure with the layers beneath. Because the solid or ordered second layer may form on either of two equivalent sublattices, there must be a phase transition, associated with the broken stacking degeneracy, separating the ordered and disordered phases. In the third layer, however, no such phase transition is expected to occur because the first layer determines a preferred sublattice for the third-layer ordered phase. Higher layers are expected to resemble the third for the same reason. ${ }^{8}$

In a recent $x$-ray study of krypton films ${ }^{9}$ by Hainsey et al. (HGSS), a multicritical point was found in place of the second-layer condensation critical point. According to this result, the first-order layering transition does not end, but continues to higher temperatures as a line of continuous transitions. At higher coverages, that study found evidence of another continuous order-disorder transition at which the second layer solidifies. HGSS interprets the region between the two transitions to be an "intermediate" disordered bilayer phase in which the disordered second layer is thought to induce disorder in the first layer. The third layering transition was found to end at a critical point in agreement with the fcc adsorption theory.

In our thermodynamic study, we bridge the gap between the ellipsometry and $x$-ray results. We confirm the reentrant layering behavior and find several additional phase transitions not observed in the ellipsometry study. In the second layer we find mesa-like heat-capacity features coinciding with the proposed disordered bilayer region. The second layering transition seems to end on a condensation critical point, but our data are not conclusive. On the other hand, we find that the third layering transition does not end but connects to a line of phase transitions that appear to join onto the first reentrant layering transition.

\section{EXPERIMENTAL DETAILS}

The experiment was performed using a homemade adiabatic scanning ratio calorimeter. ${ }^{10}$ The noise in the heat-capacity data is about $0.04 \%$ of the total sample cell heat capacity, and the reproducibility of data taken from different runs at the same coverage is better than $0.1 \%$. The substrate is Union Carbide Grafoam ${ }^{11}$ which has been prepared by outgassing at $1000^{\circ} \mathrm{C}$ until the pressure was below $10^{-6}$ torr, after an initial $1500^{\circ} \mathrm{C}$ heating in a chlorine atmosphere when it was manufactured. The adsorbate is $99.95 \%$ pure krypton gas. Heat-capacity scans were taken at 59 coverages between one and six layers, between 80 and $120 \mathrm{~K}$. A comparison thermal mass was heated with a constant heat input chosen to produce a scan rate of approximately $2 \mathrm{~K} / \mathrm{h}$. The thermal time constant of the cell is estimated to be about $3 \mathrm{~min}$, so the scan rate used yields about ten statistically independent data points per degree. We have measured peaks with a full width at half maximum of $0.2 \mathrm{~K}$ showing that tem- 
perature gradients in the sample cell are small during the scan and that the substrate is very homogeneous. Pressures were simultaneously measured during the heatcapacity scans with a 1000-torr Barocel ${ }^{12}$ capacitive manometer that is linear to within $0.03 \%$ of full scale, allowing a precise determination of the number adsorbed in the film and the chemical potential at each heat-capacity point. Temperatures were measured with a platinum thermometer read by a resistance bridge. Coverages are measured in STPCC's $\left(\mathrm{cm}^{3}\right.$ at standard temperature and pressure). A previous study ${ }^{13}$ using the same substrate determined the $\sqrt{3} \times \sqrt{3}$ commensurate coverage to be 165 STPCC's. The monolayer coverage, measured from the inflection point of a krypton isotherm at $102 \mathrm{~K}$, is 260 STPCC's.

The heat-capacity scans were performed in order of increasing coverage to keep the film on the adsorption branch of the capillary condensation hysteresis loop. ${ }^{14}$ This procedure minimizes the amount of capillary condensation in the system. Between scans, the film was dosed from a calibrated volume at room temperature by cooling the cell. The films were then annealed at $120 \mathrm{~K}$ and recooled according to a power-law temperature versus time protoco ${ }^{15}$ that is believed to aid in the formation of uniform films.

The heat capacity of the cell is typically $1-20 \%$ of the total background heat capacity made up of the sample cell and substrate. At higher temperatures where the saturated vapor pressure is high, a large part of the background-subtracted heat-capacity signal is due to desorption of the film. As the temperature increases, atoms leave the film and enter the vapor, so additional heat, equal to the heat of desorption per atom, must be put into the sample cell. The heat of desorption $q_{d}$ is defined by

$$
C_{0}=C_{\text {film }}+C_{\text {gas }}-q_{d}\left[\frac{\partial N_{\text {film }}}{\partial T}\right]_{N_{0}},
$$

where $C_{0}$ is the total measured heat capacity with the sample cell and bare substrate heat capacities subtracted, and $N_{0}$ is the total amount of krypton in the system, film plus vapor. Thus

$$
q_{d}=T\left(\frac{\partial S_{\mathrm{gas}}}{\partial N_{\mathrm{film}}}\right)_{T}-T\left(\frac{\partial S_{\mathrm{film}}}{\partial N_{\mathrm{film}}}\right)_{T},
$$

where $S_{\text {gas (film) }}$ and $N_{\text {film }}$ are the entropy of the gas (film) and the amount adsorbed. The entropy of the gas may be calculated from the virial equation of state. To calculate the film term we use the Maxwell relation

$$
\left(\frac{\partial S_{\mathrm{film}}}{\partial N_{\text {film }}}\right)_{T}=-\left(\frac{\partial \mu}{\partial T}\right)_{N_{\text {film }}} .
$$

This quantity may be calculated exactly if isotherms at closely spaced temperature are available. In the present situation, we only know $\mu$ along the experimental paths of constant $N_{0}$. We have found the approximation

$$
\left(\frac{\partial \mu}{\partial T}\right)_{N_{\text {film }}} \approx\left(\frac{\partial \mu}{\partial T}\right)_{N_{0}}
$$

to be adequate for our purpose. This approximation produced better results than the Frenke-Halsey-Hill approximation $^{16}$

$$
\left(\frac{\partial \mu}{\partial T}\right)_{N_{\text {film }}} \approx\left(\frac{d \mu_{0}(T)}{d T}\right),
$$

where $\mu_{0}$ is the bulk-saturated chemical potential.

To avoid adding noise to the desorption corrected data, the correction for each scan was fit to a polynomial over the entire temperature range. The purpose of this procedure is to subtract the large desorption background in order to present more clearly the sharp peaks due to phase transitions in the film. It should be noted that part of the film heat-capacity peaks may be due to desorption, because a phase transition in the film that results in a change in density may cause atoms to desorb. This contribution to the heat-capacity peaks is not subtracted when the correction is fit to a smooth function.

\section{RESULTS}

Figure 1 is a phase diagram based on our study showing the locations of heat-capacity peaks in $\mu$ versus $T$, displayed for clarity as $\left(\mu_{0}-\mu\right)^{-1 / 3}$ versus $T$. A similar phase diagram was found in argon on graphite in a previous study, ${ }^{5}$ but methane on graphite exhibits different behavior. ${ }^{17}$

Figure 2 shows some of the same data plotted in the number $N$ versus $T$ plane. In this figure, the ordinate represents the total number adsorbed as film and as bulk material condensed in capillaries in the substrate. At nominal coverages under two layers, the number of capillary condensed is very small, but this number increases dramatically at higher coverages. To form a film of six layers, the equivalent of about 20 layers of gas must be

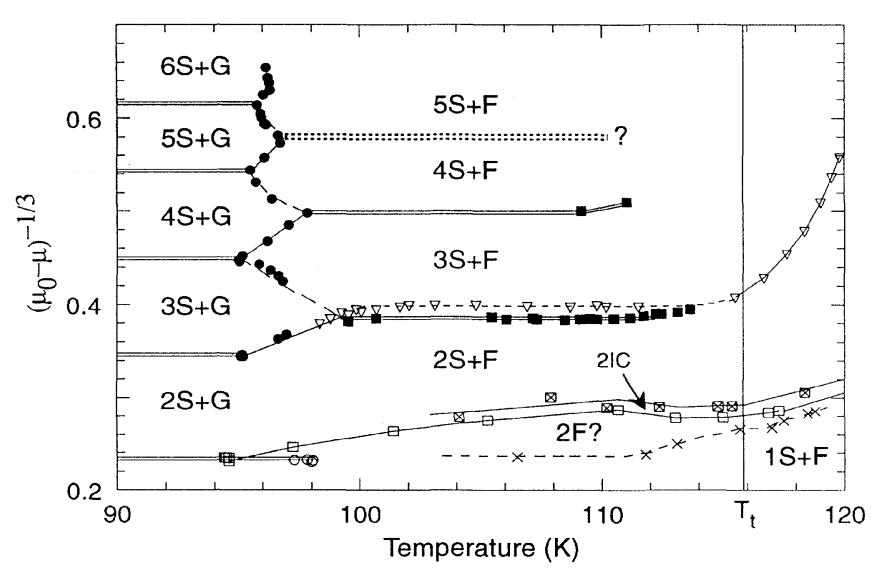

FIG. 1. Phase diagram plotted as $\left(\mu_{0}-\mu\right)^{-1 / 3}$ (in $\mathrm{K}^{-1 / 3}$ ) vs T. $N S+G$ means $N$ solid layers with some $2 \mathrm{D}$ gas adsorbed in the uppermost layer. $N S+F$ means $N$ solid layers with a dense disordered phase in the uppermost layer. $2 I C$ signifies a mutually incommensurate solid bilayer. $2 F$ is the disordered bilayer phase proposed by GHSS. Symbols are described in the text. 


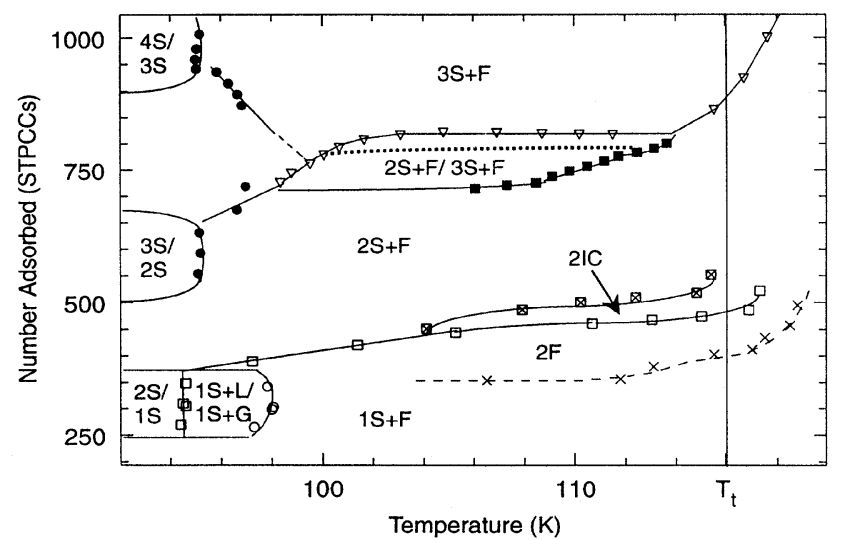

FIG. 2. Phase diagram in temperature vs total number adsorbed. Regions marked with two phases separated by a solidus represent coexistence regions. $2 S / 1 S$ means a coexistence between bilayer and monolayer solid films. $2 S+F / 3 S+F$ is the first reentrant layering transition. Other notations are the same as in Fig. 1.

adsorbed into the sample cell. Below we will present the heat-capacity evidence for the various phase transitions shown in Figs. 1 and 2.

\section{A. Second-layer phase diagram}

Representative heat-capacity scans around bilayer coverages are shown in Fig. 3. The scans for the first four

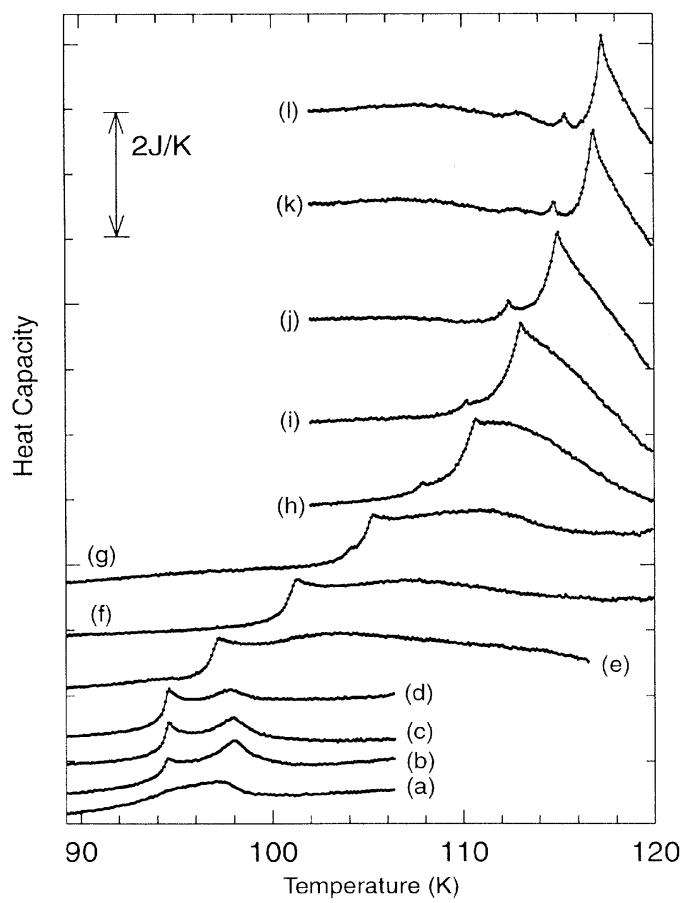

FIG. 3. Heat-capacity scans for coverages around the formation and completion of the second layer. The curves have been translated in the $y$ direction for clarity. Coverages, at the first peak for all scans are the following: (a) 270, (b) 304, (c) 309, (d) 348, (e) 398, (f) 421, (g) 444, (h) 460, (i) 468, (j) 473, (k) 486, and (1) 520 STPCC's. coverages show two features. The features at about $94 \mathrm{~K}$, denoted by open squares in Figs. 1 and 2, occur at the same temperature and chemical potential to within experimental accuracy. We interpret this point as a twodimensional (2D) triple point at which three phases, solid, liquid, and gas, coexist on top of an ordered first layer. If the transition between ordered and dense disordered phases is continuous, this phenomenon is then known as the critical end point of the order-disorder transition. The second peaks (open circles, Figs. 1 and 2) are due to crossing the boundary between twodimensional liquid-gas coexistence and uniform fluid phases. These points describe a blunt-ended coexistence region in the $N-T$ plane.

Figure 4(a) shows the experimental paths in the $\mu$ versus $T$ plane. Curves that collapse to the same chemical potential are from scans that pass through layering coexistence regions. From the peak positions plotted in
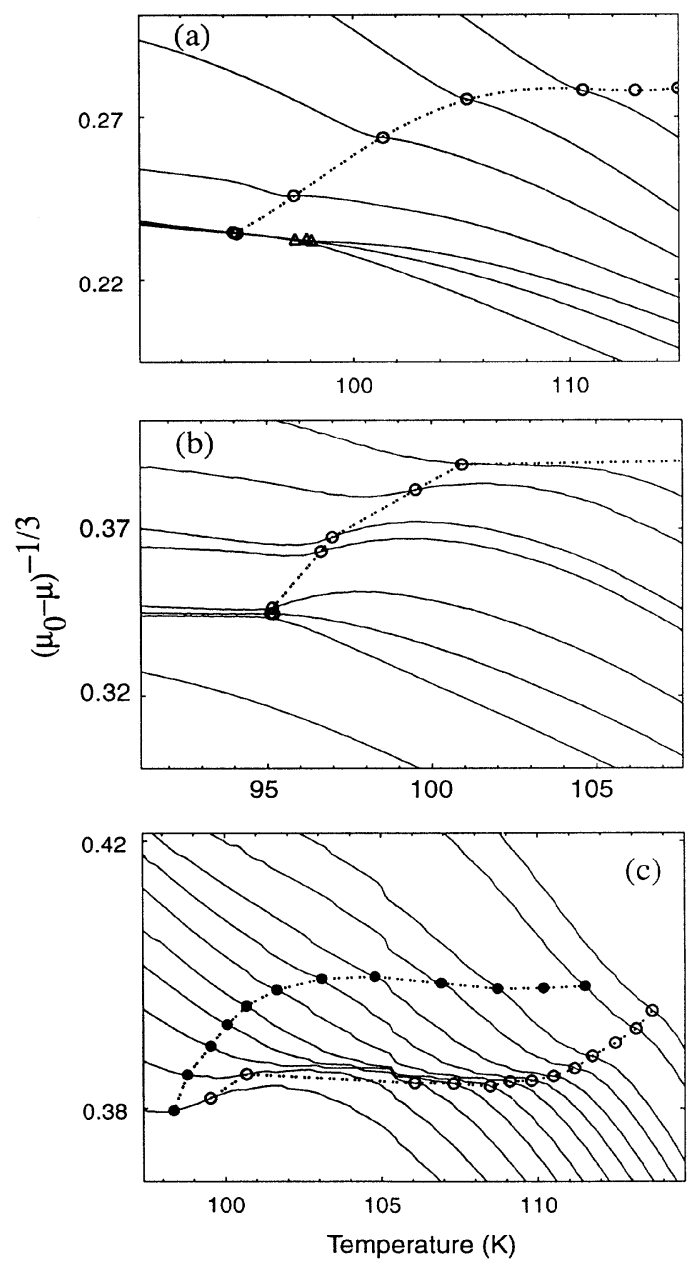

FIG. 4. Experimental trajectories in $\left(\mu_{0}-\mu\right)^{-1 / 3}$ (in $\mathrm{K}^{-1 / 3}$ ) vs $T$ around (a) the end of the second layering transition, (b) the end of the third layering transition, and (c) the first reentrant layering transition. Symbols show the positions of heatcapacity peaks. Dotted lines are proposed phase transitions. Scans that collapse in $\mu$ indicate coexistence regions. 
Fig. 4(a) (triangles), we see that the peaks occur as the scans leave the coexistence region.

Scans at higher coverages in Fig. 3 show different behavior. The heat-capacity data show a sharp peak [open squares, Figs. 1 and 2; open circles, Fig. 4(a)] followed by a broad mesa with a more gradual fall off at higher temperatures. The sharp peaks trace out a phase boundary that intersects the layering transition at the triple point. This phase transition appears to be the melting of the second layer. A point-by-point desorption correction shows that much of the heat-capacity mesa can be accounted for by desorption of the film. However, our data are not conclusive on whether the system crosses a real phase boundary near the end of the heat-capacity mesa.

The approximate positions of the falloffs of the mesas are plotted as $\times$ symbols in Figs. 1 and 2 . The location of the phase boundary that they may represent is shown with a dashed line. The region in the $\mu$ versus $T$ plane thus mapped out corresponds to the intermediate bilayer disordered region found in the x-ray study. The dashed phase boundary may intersect the layering transition at its end point or at lower temperatures. Our heat-capacity data [curves (a)-(d) in Fig. 3] resemble the signature of a 2D critical point found in other systems. ${ }^{17,18}$ However, a vapor pressure study ${ }^{19}$ found that the exponent governing the end of the second layering transition in krypton differed significantly from the expected 2D Ising value.
The fcc adsorption model $^{7}$ predicts that the phase transition separating the ordered from the disordered phase should be second order at high temperatures due to the broken stacking degeneracy. This model assumes that the second layer is commensurate with the first. From their $x$-ray data, HGSS speculate that both orderdisorder transitions found are infinite order (essential singularity) and are of the Kosterlitz-Thouless-HalperinNelson-Young (KTHNY) type. ${ }^{20}$ In this view, the region between the low-temperature (open squares, Figs. 1 and 2 ) and high-temperature ( $X$ 's, Figs. 1 and 2 ) transitions would correspond to a hexatic phase. Our results are not inconsistent with the data of Gangwar and Suter ${ }^{19}$ and HGSS, ${ }^{9}$ which show sharp behavior in the thermodynamic signal and the scattering parameters, respectively. However, we believe our data make unlikely the interpretation of the first (low-temperature) transition as infinite order, because we observe sharp heat-capacity peaks. At higher coverages, we observe the $\mu$ versus $T$ trajectories to follow the phase boundary for a short interval. This behavior may indicate that the phase transition is first order, but the data cannot be considered conclusive on this point, and it is still possible to speculate about the nature of the enclosed phase. Finally we note that at higher coverages, the first order-disorder transition is itself a two step process. We observe a small peak preceding the second-layer melting peak for all but the first two scans
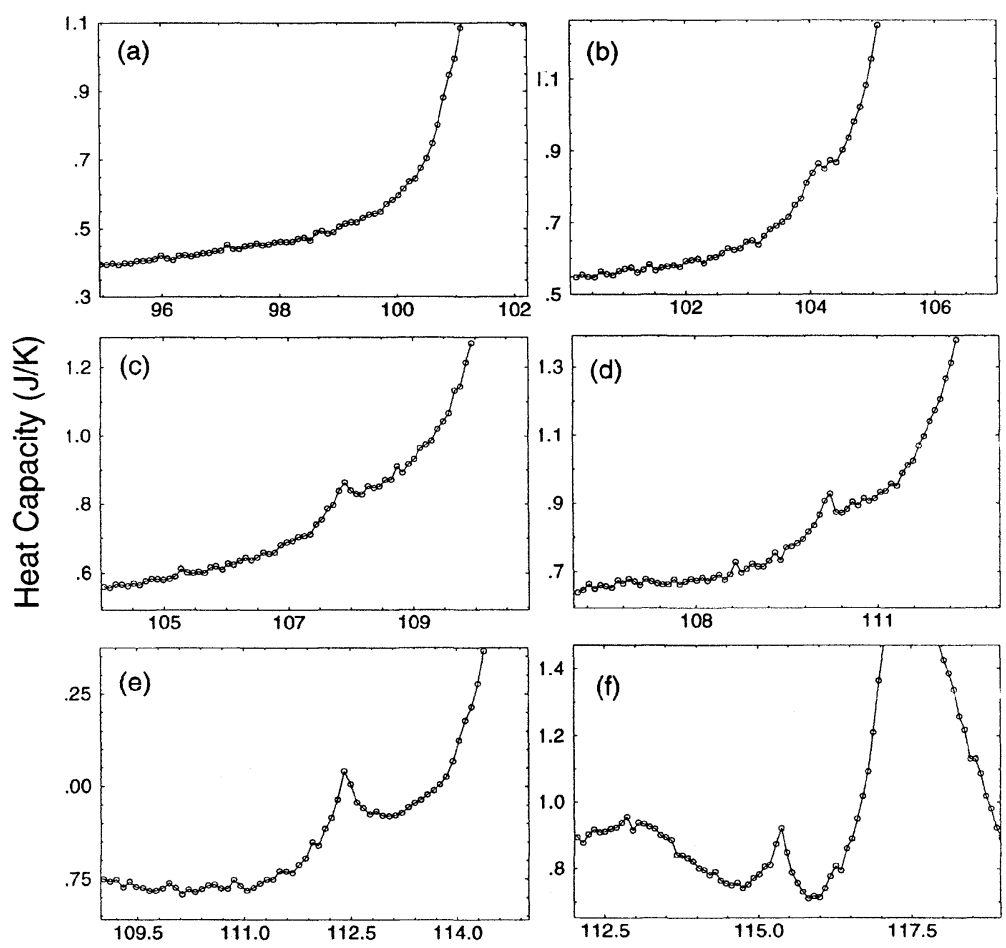

FIG. 5. Heat-capacity scans showing the rising edges of the second layer melting peaks for selected scans above the second layer triple point. Small premelting peaks (crossed squares, Figs. 1 and 2) are seen in (b)-(f). These peaks may result from a CI transition between solid first and second layers. Coverages at the melting peak are $421,444,460,468$, 473, and 520 STPCC's.

Temperature (K) 
above the $2 \mathrm{D}$ triple point pressure (Fig. 5 and crossed squares, Figs. 1 and 2). This peak is most likely due to a commensurate-incommensurate (CI) transition at which the second layer becomes expanded with respect to the first layer. This identification is made because the peak resembles those in other systems where scattering experiments have identified CI transitions. If the melting transitions take place from an incommensurate phase, then we would not expect the fcc adsorption model to apply. Rather the transition would be the melting of a 2D solid, which could be either first order or of the KTHNY type.

\section{B. Third-layer phase diagram}

Figure 6 shows heat-capacity features at around the formation and completion of the third layer (filled circles, Figs. 1 and 2). The striking difference between these peaks and those of Fig. 3 is that scans that pass along the layering transition have only one peak. The three lowest peaks in Fig. 6 occur at the same temperature and chemical potential to within the experimental accuracy. These peaks join onto a line of phase transitions extending to higher temperatures and coverages, so that the third layering transition in krypton does not appear to end at a condensation critical point. We believe that the line of peaks, represented with a dotted line in Fig. 4(b), indicates real phase transitions and not just some nonsingular behavior associated with passing close to a critical point, because we continue to observe these peaks at temperatures and coverages well above $95 \mathrm{~K}$; in fact, they extend to above $116 \mathrm{~K}$, the bulk triple point temperature. If this line of phase transitions is a second-order line, then the third layering transition ends at a tricritical point. This

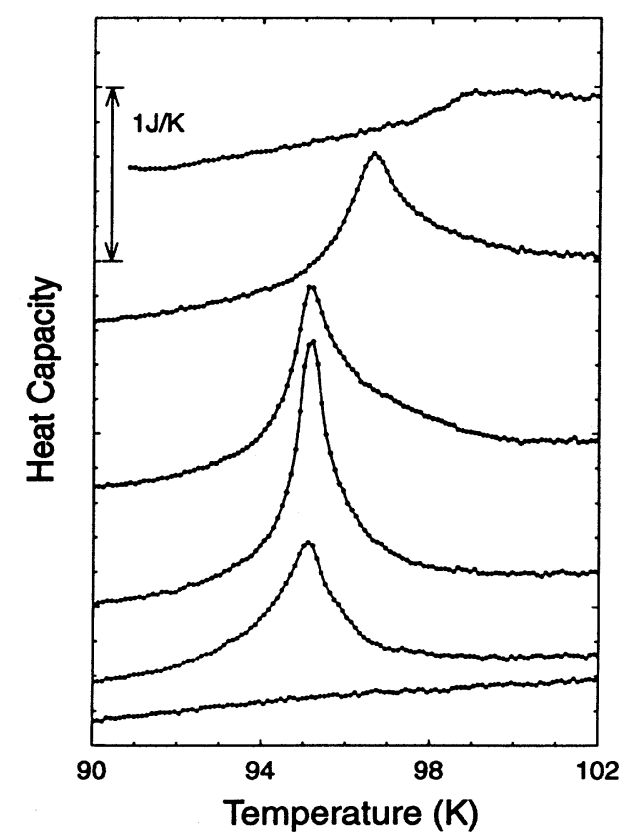

FIG. 6. Heat-capacity scans for coverages around the formation and completion of the third layer. Coverages at the peak are 553, 592, 631, 674, and 762 STPCC's. scenario might be realized if, for example, the interaction of the third-layer atoms with those of the first layer were not strong enough to determine a preferred sublattice. In this case, the third-layer phase diagram looks almost exactly like the phase diagram for the second layer of the fcc adsorption model. ${ }^{7}$ Another possibility is that the phase transition is first order and that the third-layer phase diagram follows the incipient triple point behavior found in monolayer $N_{2}{ }^{21}$

\section{Upper layers}

Figure 7 shows heat-capacity scans taken in the neighborhood of the reentrant layering transition at $3 \frac{1}{2}$ layers. The $\mu$ versus $T$ data in Fig. 4(c) clearly show that scans pass through a coexistence region. These scans show two features in the heat capacity. The first is a small peak (inverted triangles, Figs. 1 and 2) that was also seen in the corresponding layering transition in argon. There we noted that the small peak seemed to occur as the system entered the coexistence region. In krypton we have made several more scans in this region. The data show that the small peak consistently occurs slightly before the scans hit the layering transition. We now believe that this feature is not directly associated with reentrant layering but instead may be due to the melting of the third layer which is seen to continue at higher temperature (also shown with inverted triangles, Figs. 1 and 2) after the

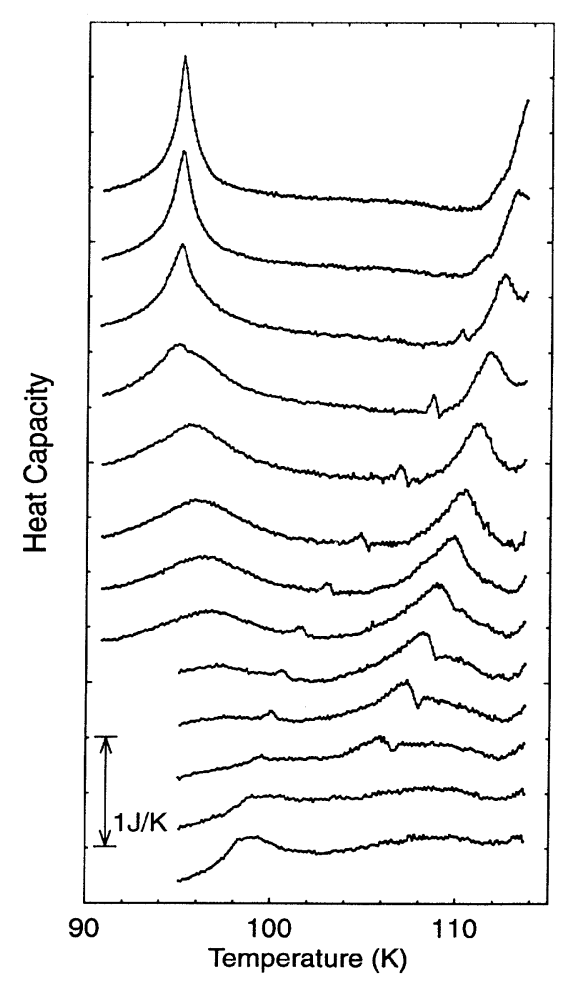

FIG. 7. Closely spaced heat-capacity scans (displaced upwards for clarity) around the first reentrant layering transition. Scans differ in coverage by about $\frac{1}{15}$ of a nominal layer. Coverages are between 726 and 820 total STPCC's adsorbed. 


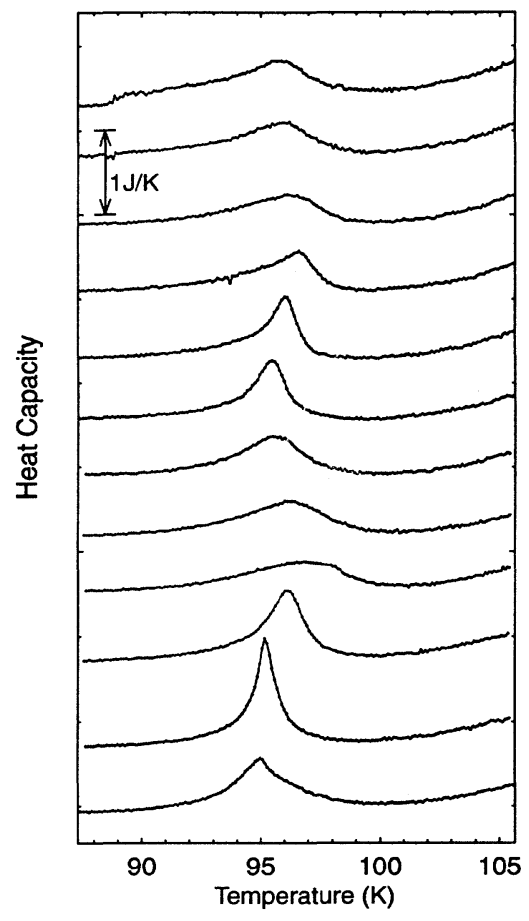

FIG. 8. Heat-capacity scans showing the low-temperature peak for coverages higher than four layers. This peak may be due to the preroughening transition. Coverages are 965, 1031, $1111,1202,1409,1557,1704,1849,2036,2211,2430$, and 2544 total STPCC's adsorbed.

reentrant layering transitions have ended.

The larger broader peaks following (filled squares, Figs. 1 and 2) are due to the system leaving the coexistence region. We no longer believe there is a heat-capacity peak where the scans enter the coexistence region, but we can estimate the temperature where each scan enters coexistence via the chemical potential data. Knowing those points we can estimate the coverage discontinuity to be less than one-half a layer. We are able to identify two peaks (not shown) at around $4 \frac{1}{2}$ layers due to the next reentrant coexistence region. These peaks are not preceded by smaller peaks, and we have made no effort to map out the phase boundary in detail. In argon, we were able to observe heat-capacity peaks for the first three reentrant layering transitions at $3 \frac{1}{2}, 4 \frac{1}{2}$, and $5 \frac{1}{2}$ layers, but the features become less noticeable with increasing coverage. We believe that the ellipsometry results ${ }^{1,3}$ give convincing proof that the reentrant transitions do exist in higher layers, but it is interesting that the heat-capacity signature disappears.

At lower temperatures, we clearly observe a series of zig-zagging peaks at all coverages (Fig. 8 and filled circles in Figs. 1 and 2). These peaks join the end points of the low-temperature layering transitions with the starting points of the reentrant layering transitions. Because the uppermost layer of the film is solid at low temperatures, the forward sloping sections of the zig zags could be either due to the disordering of the uppermost layer or, possibly, to a rapid crossover between a coverage of around $N$ layers to a coverage of around $N-\frac{1}{2}$ layers. Similarly, the backwards sloping sections may be a real phase transition between $N$ and $N+\frac{1}{2}$ layer films or may only represent a rapid crossover. A backwards sloping phase boundary indicates that both the density and the entropy are greater in the high-temperature phase.

The low-temperature peaks may be associated with a preroughening (PR) transition at the bulk surface. It has been predicted ${ }^{6}$ that these peaks should show a crossover from Ising-like behavior around the layering critical points to preroughening behavior in very thick films. The specific-heat exponent for the PR transition is predicted to vary continuously between $-\infty$ and $\frac{2}{3}$ depending on the ratio of nearest- and next-nearest-neighbor interaction energies, ${ }^{6}$ so the model does not specify the form of the heat-capacity signal.

\section{CONCLUSIONS}

We have found that the multilayer phase diagram for krypton adsorbed on graphite is very similar to that of argon on graphite. The second layer acts as an independent two-dimensional system, although it acts quite differently from monolayer krypton on graphite. ${ }^{22}$ The observation of an order-disorder transition in the second layer is consistent with the prediction of the fcc adsorption theory and with the results of an x-ray study, although the details may be different. The fourth layer acts the same as the fifth and sixth as far as we can tell from our measurements. The surface of these thicker films may approximate the behavior of the crystal interface with a possible preroughening transition and a roughening transition at higher temperature. The third layer may be viewed as a crossover region between the twodimensional behavior of the lower layers and the threedimensional interface behavior of thicker films, where more than the uppermost layer participates in phase transitions.

Clearly there is a need for more study in this system to resolve the many mysteries that remain. We especially look forward to the availability of scattering results for films of over three layers.

\section{ACKNOWLEDGMENTS}

We have enjoyed fruitful discussions with Dr. Peter Weichman and with Dr. Oscar Vilches. This work was supported by DOE Grant No. DE-FG03-85ER45192.
${ }^{1}$ G. B. Hess, in Phase Transitions in Surface Films 2, edited by H. Taub et al. (Plenum, New York, 1991).

${ }^{2}$ M. P. Nightingale, W. F. Saam, and M. Schick, Phys. Rev. Lett. 51, 1275 (1983); J. D. Weeks, Phys. Rev. B 26, 3998
(1982); D. A. Huse, ibid. 30, 1371 (1984).

${ }^{3}$ H. S. Youn and G. B. Hess, Phys. Rev. Lett. 64, 918 (1990).

${ }^{4}$ M. T. Alkhafaji and A. D. Mingone, Phys. Rev. B 45, 8767 (1992). 
${ }^{5}$ P. K. Day, M. J. Lysek, M. LaMadrid, and D. L. Goodstein, Phys. Rev. B (to be published).

${ }^{6} \mathrm{~K}$. Rommelse and M. den Nijs, Phys. Rev. Lett. 59, 2578 (1987); M. den Nijs, ibid. 64, 435 (1990); see M. den Nijs in (Ref. 1).

${ }^{7}$ W. F. Saam, Surf. Sci. 125, 253 (1983).

${ }^{8}$ The same arguments apply for other types of stacking.

${ }^{9}$ R. F. Hainsey, R. Gangwar, J. D. Shindler, and R. M. Suter, Phys. Rev. B 44, 3365 (1991).

${ }^{10}$ M. J. Lysek, P. K. Day, M. LaMadrid, and D. L. Goodstein, Rev. Sci. Instrum. 63, 5750 (1992).

${ }^{11} \mathrm{Grafoam}$ is a registered trademark of Union Carbide Corporation.

${ }^{12}$ Barocel is a trademark of Datametrics.

${ }^{13}$ M. J. Lysek, Ph.D. thesis, California Institute of Technology, 1991.

${ }^{14}$ M. J. Lysek, M. LaMadrid, P. K. Day, and D. L. Goodstein, Langmuir 8, 898 (1992).

${ }^{15}$ The temperature of the cell was controlled during the cooling cycle so that the cooling rate satisfied $|d T / d t|<C\left(t_{1}-t\right)^{3}$. Any cooling schedule that slows the cooling rate at low tem- perature where diffusion times are large should aid in the formation of uniform films. The total cooling time from 120 to $80 \mathrm{~K}$ was $12 \mathrm{~h}$.

${ }^{16}$ W. A. Steele, Films on Solid Surfaces (Academic, New York 1975), p. 238.

${ }^{17}$ M. J. Lysek, M. A. LaMadrid, P. K. Day, and D. L. Goodstein, Phys. Rev. B (to be published).

${ }^{18}$ H. K. Kim and M. H. W. Chan, Phys. Rev. Lett. 53, 170 (1984); H. K. Kim, Y. P. Feng, Q. M. Zhang, and M. H. W. Chan, Phys. Rev. B 37, 3511 (1988).

${ }^{19}$ R. Gangwar and R. M. Suter, Phys. Rev. B 42, 2711 (1990).

${ }^{20}$ D. R. Nelson and B. I. Halperin, Phys. Rev. B 19, 2457 (1979); A. P. Young, ibid. 19, 1855 (1979); J. M. Kosterlitz and D. J. Thouless, J. Phys. C 6, 1181 (1973); J. M. Kosterlitz, ibid. 7, 1046 (1974).

${ }^{21}$ M. H. W. Chan, A. D. Mingone, K. D. Miner, and Z. R. Li, Phys. Rev. B 30, 2681 (1984).

${ }^{22}$ D. M. Butler, J. A. Litzinger, A. J. Stewart, and R. B. Griffiths, Phys. Rev. Lett. 42, 1289 (1979); M. H. W. Chan, Phase Transitions in Surface Films 2 (Ref. 1), and references therein. 